the estimation of PWC and MWC parameters. Integrated indices such as BA on MWC, BA on PWC, BA on (MWC + PWC), predicted biological age (PBA) and ageing rate (BAPBA) were calculated.

Results Statistically significant differences between MWC, PWC levels and ageing rates in the studied groups were revealed $(p<0.001-0.05)$. BA indices and ageing rates of lorry-drivers were significantly higher in comparison with control group $(p<0.001)$. The results presented showed that in equal conditions of submaximum physical load in both groups a significant premature decrease in adaptation ability of lorrydrivers' cardiovascular system was observed $(p<0.001)$. Analysis of correlations between length of service and CA of lorrydrivers from one side and MWC, PWC, BA indices and ageing rates from the other showed that most of criteria under study depend on driving experience significantly more than on CA of lorry-drivers $(\mathrm{p}<0.001-0.05)$. The $40-49$ year-old lorry-drivers with 15-19 years of driving experience were identified as a risk group with the symptoms of premature ageing.

Conclusion The above studies revealed the occupational environment and long driving experience being the risk factors for the accelerated ageing of lorry-drivers, which can result in health problems, occupational and work-related diseases.

\section{NEW EVIDENCE ON CHD RISKS DUE TO PSYCHOSOCIAL STRESS AT WORK AND PHYSICAL ACTIVITY}

'Johannes Siegrist*, ${ }^{2}$ Alicja Bortkiewicz*. ${ }^{1}$ Faculty of Medicine, University of Düsseldorf, Düsseldorf, Germany; ${ }^{2}$ Department of Work Physiology and Ergonomics, Nofer Institute of Occupational Medicine, Lodz, Poland

\subsection{6/oemed-2018-ICOHabstracts. 169}

The topics of the session will cover interdisciplinary research on occupational and environmental agents (mainly stress, occupational physical activity, lifestyle) that may increase the risk of cardiovascular diseases (CVD). Those diseases are still a major health problem in Europe and, therefore, an efficient research on this topic is necessary In the frame of the session it will be also presented results of WHO-ILO review on psychosocial risk factors (especially job insecurity and long working hours) and ischaemic heart disease.

Els Clays ${ }^{1}$, Marco M Ferrario ${ }^{2}$, Giovanni Veronesi ${ }^{3}$, Johannes Siegrist ${ }^{4}$

${ }^{1}$ Department of Public health, Ghent University, Ghent, Belgium

${ }^{2}$ Research Centre in Epidemiology and Preventive Medicine, Department of Medicine and Surgery, University of Insubria at Varese, Varese, Italy

${ }^{3}$ Research Centre in Epidemiology and Preventive Medicine, Department of Medicine and Surgery, University of Insubria, Varese, Italy.

${ }^{4}$ Faculty of Medicine, University of Düsseldorf, Düsseldorf, Germany

\section{1a THE ROLE OF OCCUPATIONAL PHYSICAL ACTIVITY AND WORK STRESS IN CARDIOVASCULAR DISEASE}

E Clays. Department of Public health, Ghent University, Ghent, Belgium

10.1136/oemed-2018-ICOHabstracts.170
Introduction Growing evidence has shown that occupational physical activity increases the risk for cardiovascular disease and mortality. Performing strenuous physical activities at work remains a daily reality for a significant part of workers in different sectors. There is thus a critical need to develop preventive measures against premature cardiovascular disease risk in workers with high physical demands. It is particularly important to elucidate structural preventive measures at the collective workplace level for primary prevention of cardiovascular disease in this group. The psychosocial work stress environment offers opportunities to counter the harmful effects of physical work demands.

Methods and result An overview will be presented of available studies addressing moderating effects of psychosocial stress measures in the relation between occupational physical activity and cardiovascular disease. A limited number of studies have shown that psychosocial resources - like social support at work and level of job control - may buffer the harmful effect on health from physical work demands. In addition, this presentation will focus on the potential mechanisms by which the psychosocial work environment might play a role in the effects of occupational physical activity on cardiovascular health outcomes.

Discussion Research showing that occupational physical activity does not produce cardiovascular health benefits - like leisure time physical activity does - has rapidly expanded over the past decade. On the contrary, more and more evidence confirms that engaging in high levels of occupational physical activity generates increased risk of cardiovascular disease. One of the current challenges in this research field is to investigate how the psychosocial work environment may play a role in this relation. Empirical evidence on the buffering effect of psychosocial stress measures in the relation between occupational physical activity and cardiovascular outcomes is still quite scarce. More detailed investigations using objective measurements are needed.

\section{1b EXPLORING THE COMBINED EFFECT OF JOB STRAIN AND OCCUPATIONAL PHYSICAL ACTIVITY ON CARDIOVASCULAR DISEASE INCIDENCE}

\begin{abstract}
1,2,3 MM Ferrario*, ${ }^{2} \mathrm{G}$ Veronesi, ${ }^{2,3} \mathrm{R}$ Borchini, ${ }^{1} \mathrm{M}$ Roncaioli, ${ }^{4} \mathrm{G}$ Grassi, ${ }^{4} \mathrm{G}$ Cesana. ${ }^{1}$ School of Occupational Medicine, University of Insubria at Varese, Varese, Italy; ${ }^{2}$ Research Centre in Epidemiology and Preventive Medicine, Department of Medicine and Surgery, University of Insubria at Varese, Varese, Italy; ${ }^{3}$ Occupational Medicine Unit, Varese University Hospital, Varese; ${ }^{4}$ Department of Medicine, University of Milano-Bicocca, Monza, Italy
\end{abstract}

\subsection{6/oemed-2018-ICOHabstracts.171}

Introduction The aim of the study is to investigate the interplay between job strain (JS) and occupational physical activity (OPA) in determining the risk of major cardiovascular diseases (CVD), in a working male population.

Methods $n=1515$ participants to three population-based (WHO-MONICA Brianza II and III survey and PAMELA) North Italian cohorts, 25-64 years old, employed and CVDfree at baseline, were available for the analyses. JS was investigated using the Job Content Questionnaire (MONICA-MOPSY short version), and dichotomized as high vs no-high strain. A habitual OPA score was derived from the Baecke Questionnaire (8 items) and categorised in tertiles. Age-adjusted hazard ratios (HRs) and 95\% confidence intervals for incidence of CVD (first coronary heart disease or ischaemic stroke, fatal or non-fatal) events were estimated from Cox-proportional hazard 